\title{
BMJ Open Living labs for patient engagement and knowledge exchange: an exploratory sequential mixed methods study to develop a living lab in paediatric rehabilitation
}

\author{
Mandy M Archibald (D , ${ }^{1}$ Kristy Wittmeier, ${ }^{2}$ Matthew Gale, ${ }^{3}$ Florencia Ricci, ${ }^{2}$ \\ Kelly Russell, ${ }^{2}$ Roberta L Woodgate (D) ${ }^{1}$
}

To cite: Archibald MM, Wittmeier K, Gale M, et al. Living labs for patient engagement and knowledge exchange: an exploratory sequential mixed methods study to develop a living lab in paediatric rehabilitation. BMJ Open 2021;11:e041530. doi:10.1136/ bmjopen-2020-041530

- Prepublication history for this paper is available online. To view these files, please visit the journal online ().

Received 10 June 2020 Revised 14 April 2021 Accepted 15 April 2021

Check for updates

(c) Author(s) (or their employer(s)) 2021. Re-use permitted under CC BY-NC. No commercial re-use. See rights and permissions. Published by BMJ.

${ }^{1}$ College of Nursing, University of Manitoba, Winnipeg, Manitoba, Canada

${ }^{2}$ Pediatrics and Child Health, University of Manitoba, Winnipeg, Manitoba, Canada ${ }^{3}$ Rehabilitation Centre for Children, Winnipeg, Manitoba, Canada

Correspondence to Dr Mandy M Archibald; mandy.archibald@umanitoba.ca

\section{ABSTRACT}

Introduction Despite recognition of the importance of patient engagement in research and knowledge translation, systematic approaches to engagement and co-ideation remain limited. Living labs are collaborative knowledge sharing systems that use multimethod, usercentred approaches that hold potential to catalyse these aims. However, their use in healthcare is limited, and no living lab has been developed in paediatric rehabilitation. In response to this gap and to propel innovative knowledge exchange, we propose a mixed methods study to codevelop a living lab prototype (ie, preliminary infrastructure with opportunity for scale up) in paediatric rehabilitation, with relevance to other healthcare contexts.

Methods An exploratory sequential mixed methods study will be undertaken to determine research and knowledge exchange priorities and to inform the development of the living lab prototype. Stage 1: we will use a multipronged approach to sample 18-21 youth with developmental differences or rehabilitation needs, their youth siblings and parents/guardians from a provincial paediatric rehabilitation centre, to participate in qualitative and arts-based data collection. Data will provide insight into desirable features of the living lab. Stage 2: E-surveys to youth, siblings, parents/guardians and clinicians who receive or provide services at this same centre will expand on priorities and living lab features. Stage 3 : integrated analysis will inform the living lab prototype development.

Analysis Inductive thematic analysis using interpretive description, integrated analysis of visual data and descriptive and content analysis of e-survey data will be undertaken. Joint displays will facilitate data integration. Priorities will be identified using a modified rank-order method for each key living lab domain.

Ethics and dissemination Institutional ethics and site approval have been granted. A parent advisory group and rehabilitation engineering partners will confer on data and inform the development of the living lab prototype. User engagement with the prototype will occur during an online or in-person event, and findings shared through non-technical research summaries, journal articles and academic presentations.
Strengths and limitations of this study

- This is the first study using co-design to develop a living lab for improved knowledge exchange practices in paediatric rehabilitation.

- This study uses an innovative mixed methods approach incorporating visual data, thereby increasing the relevancy of the living lab development to participants' experiences.

- The study design is focused on early stakeholder engagement and guidance for co-designing a systematic approach to innovation rooted in user needs.

- Incorporating multiple stakeholders including youth, their siblings, parents/guardians and health professionals will enable a range of perspectives pertinent to the usability and relevancy of the living lab.

- The primary limitation of this study is that data will be gathered from a single site in a metropolitan centre, limiting generalisability of the results.

\section{INTRODUCTION}

Prominent in 21st century western health discourse are persuasive narratives of patient engagement and knowledge translation (KT). Patient engagement involves the meaningful collaboration of research participants as partners in research, while KT emphasises the synthesis, dissemination, application and exchange of knowledge to inform healthcare practice and policy. ${ }^{1}$ Underlying these concepts is the understanding that research evidence produced through patient-engaged strategies will be more relevant to knowledge users, better aligned with stakeholder priorities, more readily implemented and more likely to improve the patient experience of healthcare..$^{2-5}$

Although its importance has long been recognised, patient-engagement approaches often are limited to consultation ${ }^{26}$ and fail 
to maximise the full benefits of true patient engagement at the health system (eg, appropriate use of services), personal (eg, satisfaction with services), public (eg, improved health) and societal (eg, improved social cohesion) levels. ${ }^{5}$ Achieving authentic and sustainable patient and family engagement may require new approaches which privilege inclusivity and lessen over-reliance on traditional engagement methods (eg, privileging written text) and traditionally represented groups (eg, adults).$^{7-9}$ In the contexts of paediatric rehabilitation and disability research, a consequence of these practices has been the exclusion of the voices of youth with developmental differences and their siblings, which risks further inequity and exclusion. ${ }^{9} 10$ New sustainable engagement approaches are needed to create meaningful spaces for knowledge exchange-spaces that reflect the experiential knowledge of stakeholders. Merging engagement and knowledge exchange in such a manner could facilitate understanding of stakeholder experiences and iteratively inform research and clinical care tailored to stakeholder priorities. Living labs provide an emerging opportunity to catalyse such objectives.

\section{Living Labs}

Living labs are open systems of knowledge exchange, co-ideation and testing that occur between diverse stakeholder groups in real-life settings. ${ }^{11-14}$ A systematic review of the living lab literature demonstrated a marked increase in interest in living labs since 2015, evidence of living labs as a 'multidisciplinary phenomenon', ${ }^{14}$ (p. 976) and identified a set of features and principles characteristic of living labs. ${ }^{14}$ These included a technological infrastructure, a stakeholder ecosystem, community involvement and positioning the living lab in the natural environment of the user. ${ }^{14-16}$ Similarly, the Living Lab Methodology Handbook ${ }^{17}$ identifies the following five common elements of (1) multimethod approaches, (2) user engagement, (3) multiple stakeholders, (4) real-life settings and (5) co-created environment for innovation as key to the living lab approach. ${ }^{18}{ }^{19} \mathrm{~A}$ characteristic shift towards the user suggests a high relevancy of living labs to integrated KT (ie, KT involving stakeholders throughout the research process), by way of a systematic knowledge exchange platform. Yet, reviews of living labs in other sectors (eg, information and communication technology) demonstrate an under emphasis on this co-ideation or context analysis as a key aspect of innovation and development. ${ }^{20}$

Despite the potential of living labs to promote collaborative ideation and knowledge exchange, their potential within healthcare has yet to be fully realised. ${ }^{11-13}$ While an integrative literature review of living labs in health ${ }^{18}$ identified that approximately $52 \%$ of living labs activity involve health and well-being, we have observed few published studies on this topic. This may reflect what Schuurman and colleagues ${ }^{21}$ recognise as a case of practice outpacing theory. Existing studies focus almost predominantly on the ageing sector, with attention as well to chronic disease management. ${ }^{18}$ What is also notable is that diverse conceptualisations and hence, diverse applications of living lab concepts exist, resulting in a range of examples that to varying extents, exemplify the five common elements of living labs.

For example, Noublanche and colleauges ${ }^{22}$ sought to improve gerontechnological innovation in hospitals through use of a living lab. The team brought together technology developers and the users of the technology using 'idea incubator workshops' intended to foster co-ideation and development of new technologies in the ageing sector. Vallentin-Holbech and colleagues ${ }^{23}$ emphasised the 'real-world', multistakeholder involvement and co-creation characteristics of living labs to develop a gamified virtual reality simulation to explore youth alcohol consumption in the context of health promotion. In contrast to these co-created and user-centred environments, other uses of living labs are characteristically narrower, emphasising the technological testing and/ or continuous patient monitoring through technology use (ie, generating data in a living system). The latter has often emphasised more controlled living laboratory settings. Here for instance, Boman and colleagues ${ }^{24}$ used a living lab, built as a self-contained apartment, to conduct usability testing of a videophone for persons with dementia using observation and interview methods. While testing technology is certainly a viable application of living labs, living labs are not exclusively test beds for technology; incorporating users as co-designers is also a central aim. ${ }^{25} 26$

To these ends, there is notable potential to develop the living lab concept in paediatric contexts in consideration of improved knowledge exchange and collaborative involvement, but we are unaware of any such living lab.

Although the evidence base for living labs is growing, ${ }^{14} 1821$ systematic methods pertaining to their development, implementation and evaluation in healthcare are generally lacking. This protocol reflects our first step towards addressing these gaps, which are more specifically understood as the relatively narrow spectrum of engagement approaches utilized; the relatively nascent status of living labs in healthcare and research, particularly those targeting improved knowledge exchange and KT more generally; and that no living lab exists in paediatric healthcare to our knowledge. This work seeks to culminate in a novel knowledge generation exchange and mobilisation paradigm for paediatric rehabilitation research in the form of a clinically situated living lab. As one of the first living labs of its nature worldwide and the first focused on paediatric rehabilitation, the research proposed through this protocol could provide the framework for future co-designed living labs in other populations and jurisdictions interested in leveraging patient engagement and bidirectional information sharing at the nexus of family-centred knowledge exchange and inclusive patient engagement. 


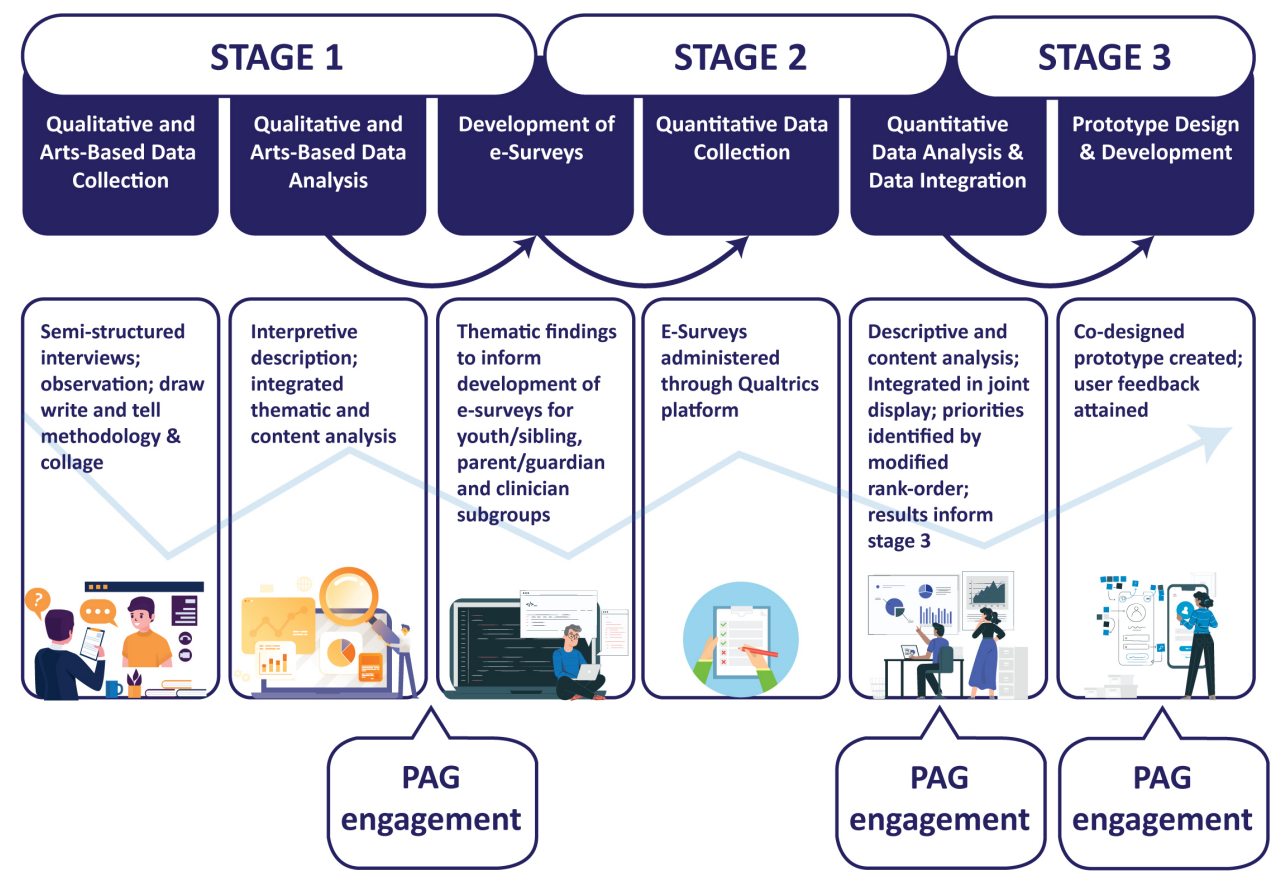

Figure 1 Study design. Visual depiction of the exploratory sequential mixed methods research design. PAG:parent advisory group.

\section{Study purpose and objectives}

The aims of this study are to (1) identify the knowledge exchange priorities (eg, methods of knowledge exchange, such as storytelling, video, real-time discussion and general priorities for learning and integrated KT) of youth with developmental differences receiving services at the paediatric rehabilitation facility, their siblings and parents/guardians, as well as direct-service clinicians in order to (2) co-design living lab prototype in a paediatric rehabilitation centre to support family-centred, inclusive and sustained knowledge exchange using inclusive and creative methodologies. The living lab prototype will be considered a proof-of-concept capable of informing future living lab implementation, evaluation and scale up within and beyond paediatric rehabilitation settings.

\section{METHODS}

\section{Design}

We will conduct an exploratory sequential mixed methods research ${ }^{27}$ design consisting of three stages: 1 . qualitative and arts-based data collection, 2. quantitative data collection and 3. living lab prototype development (figure 1). Stage 1 will involve narrative interviews guided by the interpretive description ${ }^{28}$ approach, alongside arts-based data collection and elicitation methods, using the drawwrite-tell methodology ${ }^{29}$ and collage. Analysis of stage 1 data will inform the development of the stage 2 e-survey, designed to confirm qualitative findings. Integrated data from stages 1 and 2, alongside additional insights gleaned through engagement with a parent advisory group (PAG) will inform the development of the living lab prototype, which will be unveiled for public engagement.

\section{Theoretical and conceptual framework}

The theoretical and conceptual frameworks propelling this project are the collaborative KT $(\mathrm{Co}-\mathrm{KT})^{30}$ and the Canadian Institutes of Health Research Knowledge-toAction Ethics (KTA-E) ${ }^{10}$ frameworks. The Co-KT framework was developed to facilitate collaborative knowledge production between researchers and communities. ${ }^{30}$ From this framework, we seek to enable continued dialogue between diverse stakeholders aimed at the co-creation, refinement, implementation of knowledge sensitive to local context, including methods for knowledge exchange (eg, storytelling, infographics, videobased information exchange) and general KT priorities (eg, priority learning areas, ability of living lab to enable integrated KT). The KTA-E framework was developed to illustrate ethical considerations that occur throughout the knowledge creation and KT cycle. ${ }^{10}$ From the KTA-E framework, we acknowledge the often-under recognised ethical aspects of knowledge creation and application. We aim to increase the sensitivity of knowledge exchange in paediatric rehabilitation to improve inclusiveness and collaboration with a cohort who has often experienced exclusion and oppression.

\section{Setting of study}

The study will take place at a major paediatric outpatient rehabilitation facility, located in an urban setting (population in census metropolitan area, 844 600) in central Canada. This rehabilitation facility has an interdisciplinary network of over 250 staff, with co-located partners crossing three provincial government departments. A strong culture of family engagement in health service delivery exists within this site, including the existence of a 
parent advisory committee, which collaborated to design the facility building and has identified improved research exchange and patient engagement as priorities. A rehabilitation engineering department has also been collocated within the building. The rehabilitation engineering team uses various digital and hardware technologies to create and tailor supportive devices for youth, and along with management, committed to identifying contextspecific resources and consider integration with existing structures to support future living lab feasibility and site sustainability. The unique perspectives of rehabilitation engineers who are engaged in day-to-day adaptations for youth will provide important insights into acceptable modalities and platforms for youth engagement with the future living lab.

\section{Participants}

Study participants include (1) youth, aged 5-19, with developmental differences who access services at the study site (ie, any programme and any frequency); (2) parents or guardian of youth receiving services at the study site; (3) siblings of youth receiving services at the facility and (4) clinicians who provide care for the families attending the study site. Youth at this facility receive services for a range of developmental and rehabilitation support and diagnostic needs (eg, autism spectrum disorder, global developmental delay, cerebral palsy); many youth, siblings and parents or guardians also access social and recreational services offered through the centre (eg, sibling group, dance class). Broad inclusion criteria were used to mirror inclusive study principles and ensure representation of diverse voices in the lab development and eventual use. These groups were identified as the predominant stakeholders for which the living lab prototype will be developed. English speaking youth and siblings, and parents/guardians, as well as English speaking clinicians with a professional healthcare designation will be eligible to participate in the study. We will also convene a PAG of three purposively selected parents of youth attending the study site for services. The PAG will meet three times during the study duration of 12 months to inform document development, provide feedback on emerging results and inform co-development of the living lab prototypes.

\section{Sample size and recruitment}

Stage 1

Iterative data collection and analysis is a principle guiding inductive qualitative research and will determine the precise number of participants for stage 1 . Based on our previous research and the research objectives, we anticipate approximately 18-21 participants will be suitable to achieve thematically rich data across youth, sibling and parental/guardian subgroups. Participant recruitment will be facilitated through the study site by way of introductory letters distributed to families by clinic clerks, posters with contact information, facility newsletter and social media messaging. If unsuccessful, we will engage clerks and/or clinicians to contact parents awaiting rehabilitation services at the study site. If interested in the study, a member of the research team will then approach consenting parents, to discuss the study at a time convenient to the family. Consent and assent may then be attained in a private room at the facility. If parents/guardians are unsure or if time does not permit, the family will be provided the consent-to-contact form. The consent-tocontact form will allow the research personnel to contact the family at a later date and provide more information regarding the study. If additional eligible families express interest in the study after theoretical saturation has been reached, they will be informed about the second stage of the study as an alternative option to participate.

\section{Stage 2}

A convenience sample of 192 participants (ie, 64 parents/ guardians, 64 youth and 64 siblings of the reliable selfreport age of 5 years or older) will be recruited in the facility waiting areas and over email (ie, clinicians). Sample size calculation was based on $90 \% \mathrm{CI}$, a $10 \%$ margin of error and a presenting body of approximately 1000 families to the study site per annum, and in recognition of reduced clinical intake and in-person service secondary to the COVID-19 pandemic that may influence feasible recruitment. Clerical staff will approach participants using a pre-defined script, in order to receive consent to approach before, during or after appointments. Following this approval, a research team member will then provide the study description, and attain assent and consent. If individuals are unsure or if time does not permit, the family will be provided with a consent-to-contact form. Parents will also be provided an information sheet with the research team contact information and information about the study. For the clinician sample, an email survey will be distributed to the clinical staff on the research team's behalf to the $>100$ facility-based clinicians who provide direct care for families. The email will include a brief introduction, the informed consent and e-surveyclinician version. We will use evidence-based practices to improve response rate, such as short survey length, ${ }^{31}$ clinical relevance ${ }^{32}$ and the use of email reminders-sent at 2 weeks and 4 weeks-to encourage reply from survey non-responders. ${ }^{33} 34$

\section{Data collection}

\section{Stage 1}

We will use a combination of individual semistructured interviews; observation; and the arts based data collection techniques of draw-write-tell ${ }^{29}$ and collage (for youth and siblings only), depending on the needs, preferences and ability of participants. Questions for the parent/ guardian interviews will proceed from general to specific, focusing on experiences (eg, can you tell us about your past experiences with research?), meaning (eg, would you like to be more involved in research, and if so, how?) and priorities areas for knowledge exchange and general research domains based on past experiences (eg, is there 
any information about your child's condition that would have been helpful for you? Is there a topic that you would like to learn more about?); preferences for learning and information exchange including methods and modalities (eg, how would you like to receive and share information? Storytelling, written information, video-based and so on), and concepts related to living lab development (eg, purpose, location, features).

With youth and siblings, we will offer either paper (plain or coloured A4) or an iPAD tablet for the draw-writetell approach. The option to complete a collage rather than participate in the draw-write-tell will be available for participants; family members may assist the child with the collage as necessary. The draw-write-tell technique ${ }^{29}$ was developed as a modification to the frequently used draw-and-write approach, ${ }^{35}$ which incorporates a drawing activity alongside written words produced by the participant. Draw-write-tell modifies this approach by more clearly delineating how the context of the research is explained, by not arbitrarily limiting the time or materials for art completion, by following the draw-write with a 'tell' component wherein participants interpret their own artwork, and critically, by incorporating a 'commentary' which ensures that the participant's interpretation of the artwork is privileged, and that each subdata source (draw, write, tell) is handled as an integrated unit of analysis. ${ }^{29}$

Using draw-write-tell, youth and siblings-either individually or together depending on their preferences-will be shown a series of 3-4 simple images that set the context for the research. The context for this project will include what research is and why it matters; why youth participation is important to research and healthcare; and the idea of a living lab to share information. The researcher will show the first image and read a brief introductory story about what research is and why it matters ( 85 words, or four short sentences). Participants will then be asked to complete the story through their drawing focusing on the question if we were going to create a place to share ideas about research, what would it look like, what would it focus on, and what would it be able to do?' If able, participants will be encouraged to record any words that correspond with their picture. No time limit will be set but participants will be observed and asked whether the drawing is completed. Following completion of the drawing, participants will be asked to describe the meaning of the drawing - the tell component. Here, the researcher will make a positive comment about the drawing, and start with an open ended question (ie, 'can you tell me about your drawing?'). This general question will be followed by prompting questions into certain sections of the drawing; possible ways of participating, learning and sharing within the living lab represented in the drawing or generally and possible topics that this information sharing could focus on, for example. Additional follow-up questions will focus on what research means to participants, previous experience(s) with research, how research can help them, how they would like to learn about research, what topics of research they think are important, where they would like to see the living lab located at the rehabilitation facility and any concerns, for example. If draw-write-tell is not desirable or appropriate for participants, youth and siblings will have the option to complete either a paper or iPAD tablet based collage, or merely complete a brief interview. When completed on the iPAD tablet, participants will have access to a range of icons and images pertinent to the research objectives. When completed on paper, participants will be provided with a multitude of shapes, colours and images-both representational and abstract—and asked to discuss what these meant and why they were chosen, in a manner similar to the draw-writetell question approach. Data collection is anticipated to take approximately $60 \mathrm{~min}$ per participant. Although participants' artwork will be available to the research team for analysis, participants may keep the original artwork (ie, researchers will photograph the artwork and return the original).

All interviews will be conducted at a place and time convenient to the participants, such as their home or the rehabilitation facility. Up to two experienced researchers will lead the interviews, take field notes and record observations of youth engagement, responses and family interaction. Each participant will complete a brief sociodemographic form on paper. Each participant will receive a $\$ 50$ reimbursement for participation. Of note, with the advent of the global pandemic COVID-19 that requires social distancing and reconsideration of face-to-face research methods, we may use the video-conferencing platform Zoom, which we have used with success in previous work. ${ }^{36}$

\section{Stage 2}

Thematic findings from stage 1 , clinical team expertise and PAG feedback will inform the development of three versions of an e-survey ((1) youth and sibling, (2) parent/guardian, (3) clinician). Generally, e-survey questions will mirror the domains of inquiry reflected in the stage 1 interviews as described above, enabling data convergence or divergence during mixed methods analysis. Qualitative data from stage 1 will be mined for possible response domains to populate the survey questions. For instance, youth and siblings will be asked what they would like to learn about, and how they would like to share information through the lab. The response options, such as learning about: diagnosis, how to talk with others at school or how to communicate with siblings or other family members, will originate from the qualitative data. Similarly, responses to the latter question regarding information sharing, such as writing, drawing and 'telling my story' for example, will be derived from those ideas presented by youth and siblings in the stage 1 qualitative study. Questions in the parent/guardian survey will also centre on priorities (eg, how important is it to you that you contribute to educational items for: schools? Other families? Healthcare professionals?), and desirability (eg, How desirable is it that you receive information through the following: Reading? Animated video? Discussion forums? 
Or, how desirable is it to share your experiences and ideas using: Video recording? Short-answer responses? Photographs?). Items not captured in the qualitative data but recognised as important to the research team and PAG will also be considered. E-surveys will be pilot tested with a purposive sample of three to five individuals per group (ie, youth, siblings, parents/caregivers, clinicians). Pilot e-surveys will be completed, feedback requested and surveys revised accordingly. E-surveys will be iPad hosted, password encrypted and administered using the Qualtrics platform. Clinicians will receive the e-survey through the staff email list. Youth, siblings and parents/guardians will have the e-survey presented to them by a trained member of the research team in the waiting room.

The e-surveys will include brief demographic questions for each group, followed by a series of questions centred on research priorities, KT methods and features of the living lab. Generally, the e-surveys will begin by situating participants around differing research priorities in regard to their child's conditions, emotional support, self care, medical and social needs, challenges and strengths. Questions will be asked regarding preferences in receiving and exchanging information; learning methods pertaining to coping, support, communicating, connecting and relationships for example; and how participants' experiences could be shared, translated and disseminated (eg, using methods such as animation, virtual reality or storytelling for example). Each individual will receive a $\$ 10$ gift certificate for participating in the e-survey, either at time of consent, or through an email-link for redemption.

\section{Data analysis}

Stage 1

Interviews will be digitally recorded, and transcribed verbatim using a professional transcription company. Data will be cleaned using concurrent audio playback and reading of raw transcripts to gain familiarity with the whole. Referencing of visual data will occur and preliminary analytic memos will be made. Narrative data will be iteratively and inductively analysed using the applied qualitative methodology of interpretive description ${ }^{28}$ and managed using MAXQDA software. Narrative interviews (ie, parents/guardians) will be coded line by line to determine a preliminary coding structure, accompanied by field notes and analytic memos. This approach will be completed by two members of the investigative team for the first two interviews in order to devise a preliminary coding framework. This framework will be applied to subsequent interviews, and modified according to new codes identified. Codes will be loosely grouped into categories and themes, with orientation to descriptive themes that supersede categorical containment based on the research objectives (eg, theme 1: living labs). Data from the draw-write-tell methodology will involve the construction of a 'commentary' using all three data streams (artwork, written, verbal) to triangulate the meaning of artwork and encourage holistic handling of data. ${ }^{29}$ Building off previous work conducted by the lead author $^{37}$ and others, ${ }^{38}$ arts-based data attained wherein verbal descriptions are not provided will be content analysed using a visual-coding framework to determine core constituent elements (eg, structural components), configuration (eg, of components), size (eg, emphasis), function (eg, purpose, activities) and colour (eg, suggested mood) of the living lab. ${ }^{3738}$

\section{Stage 2}

Following pilot-testing and refinement, e-survey data will be descriptively and content analysed to determine research, exchange and living lab priorities for each subgroup. Quantitative data will be considered in reference to stage 1 findings and integrated using joint displays, ${ }^{39}$ to substantiate priorities and inform the development of living lab prototype. Open-ended responses will be content analysed with a framework informed from stage 1 categories. Priorities will be identified and consolidated using a modified rank-order method ${ }^{40}$ where each participant's core priority is tabulated and weighted in relation to participants' ranking of other priorities, to produce a consolidated composite ranking for each key living lab domain. Constant comparison of themes will identify similarities and differences priorities and responses between participant groups.

\section{Prototype engagement workshop}

Stage 3

Following analysis of stage 1 and 2 data, we will convene with our PAG and clinical partners, including facility rehabilitation engineers who will develop the technological basis for the living lab system. We will collectively confer on the aims, configurations, locations and functions of the living lab prototype. We will host a half day workshop with PAG and research team members, and offer an open engagement event to attain additional input into the living lab, congruent with the co-design principles (eg, technological infrastructure, multimethod approaches). ${ }^{14}{ }^{17} \mathrm{We}$ will aim to develop a living lab prototype (ie, a basic infrastructure with capacity for scale up), in alignment with the KTA- $\mathrm{E}^{10}$ and Co-KT ${ }^{30}$ frameworks underpinning this work. Although the precise execution of the living lab prototype will be informed by stage 1 and 2 data, PAG feedback, research team input and resource considerations (eg, finances, timely updates of the content), the prototype will meet the five common elements of living labs, including use of multimethod approaches, user engagement, multiple stakeholders, real-life settings and co-created environment for innovation. ${ }^{17}$ Engagement with the living lab prototype will be facilitated through a promoted event (in-person or virtual) for users; commentary on the living lab will inform its refinement and help inform future directions for research.

\section{Patient and public involvement}

This research is supported by a PAG, which will provide input throughout each stage of the research. This PAG will meet three times throughout the study. A parent 
co-researcher is involved in the project, and early site engagement was undertaken to ensure project relevancy. Public engagement with the living lab prototype will inform further refinement and development of the living lab in the rehabilitation facility.

\section{ETHICS AND DISSEMINATION}

The Education/Nursing Research Ethics Board at the University of Manitoba approved this study. Site access approval was granted from the facility. Public engagement with the living lab prototype will be facilitated through a promoted event; commentary on the prototype will inform refinement. Participants will receive a brief, non-technical summary of the research results available during and after the event, in addition to academic publications, presentations, social media dissemination of the facility, affiliated university and investigative team.

\section{DISCUSSION}

Living labs are gaining traction in social, civil and business development circles but their potential, particularly as a knowledge exchange platform in healthcare, has not been fully realised. The current, persistent and likely steadfast decrees of participant engagement and KT mandate that researchers and clinicians reconsider the current structures and approaches available to achieve these aims. ${ }^{5}$ While not commonly conceptualised as a KT initiative, the underlying principles of living labs suggest a high level of compatibility with KT, particularly when conceptualised as an integrated, collaborative, non-linear or complex network of knowledge exchange processes involving diverse stakeholders. ${ }^{41}$ Living labs oriented towards the identification of research and selfmanagement priorities, and the collaborative ideation around co-located problems and solutions offers an innovative approach to targeting the problematic criticisms and challenges facing KT science (eg, research waste; 17-year lag times from research to practice; low resonance of KT tools with lived experience). ${ }^{42} 43$

Establishing the structure for sustained collaborative ideation to support knowledge exchange and patient engagement efforts is a formidable challenge in healthcare. As explicated by the Living Lab Methodology Handbook ${ }^{17}$ and existing reviews on living labs more broadly, ${ }^{14} 182021$ a technological infrastructure is a predominant and necessary characteristic of any living lab. The proposed research attends to this consideration through engagement with an on-site rehabilitation engineer who is embedded in the clinical environment and integrated into existing technological systems. Through this collaboration, we will leverage context-specific infrastructure, needs and institutional knowledge to produce a technological platform driving the creation of a living lab prototype (eg, a platform or infrastructure template that will require further infrastructure development to operationalize and ensure sustainable and broad use to achieve its full potential and objectives) with the purview that these systems will be better integrated, more fit for purpose and more sustainable than a top-down alternative. Producing and providing the needed proof-ofconcept for this innovative approach is a critical step towards the full-scale development, implementation and evaluation of a clinical living lab in paediatric rehabilitation, and can help provide a roadmap for development of living labs in other clinical contexts.

\section{Limitations}

This study is designed to elicit feedback to inform the development of a living lab prototype centred on user priorities at one metropolitan centre and may not be reflective of priorities of families and clinicians elsewhere. We are using a sequential design; as such, the stage 2 data collection methods are influenced by the quality of data and responses attained from stage 1 . Taking an arts-based approach to data collection with youth and siblings in stage 1 is an inclusive and developmentally sound method but is also contingent on the developmental and personal attributes of youth participants.

Twitter Mandy M Archibald @Mandy_Archibald and Roberta L Woodgate @ WoodgateRoberta

Acknowledgements We gratefully acknowledge the support from the rehabilitation service centre, including in-kind rehabilitation engineering services committed for execution of the proposed study. We acknowledge the input of CC obtained during meetings pertaining to this project, and SW for her editorial support on this manuscript. RLW is supported by a Tier 1 Canadian Research Chair (CRC) in Child and Family Engagement in Health Research and Healthcare (CIHR-Canadian Research Chair-950-231845). KW is supported by the Dr John M Bowman Chair in Pediatrics and Child Health (Endowed Chair, Rh Institute Foundation and University of Manitoba).

Collaborators Barb, Borton, Rehabilitation Centre for Children, Specialized Services for Children and Youth (SSCY) Centre.

Contributors MA conceptualised the study including leading the associated grant and writing the protocol manuscript. KW, FR, MG, KR and RLW provided meaningful contributions to aspects of study conception, the protocol draft, approved the submitted protocol and agree to be accountable for all aspects of the study described here.

Funding We would like to thank the Rady Faculty of Health Sciences (Rady Innovation Fund, Grant number 52349) for providing the funding for this research. Funders provided financial support for this forthcoming research but are not and will not be involved in the conducting or how the research is conducted.

Competing interests FR and KW are both clinicians who provide care at the rehabilitation facility. As such, there is a chance that some of the participants will recognise these names on the information sheet provided. However, these investigators will not be the individuals approaching patients, families or clinicians. These investigators will not seek consent from prospective participants at any stage of the project. These investigators will only have access to the anonymised versions of the transcripts and other data. Participants will not be penalised in any way for deciding not to participate in the study, or dropping out of the study at any time. The study is being led by MA, who declares she does not have any competing interests. MA is an applied research scientist interested in technological and methodological innovations to support knowledge exchange between diverse stakeholders, often using arts-based and mixed methods research approaches and methods of application focused on the integration of lived experience research with and evidence of effectiveness to reduce evidence misalignments.

Patient and public involvement Patients and/or the public were involved in the design, or conduct, or reporting, or dissemination plans of this research. Refer to the Methods section for further details.

Patient consent for publication Not required. 
Provenance and peer review Not commissioned; externally peer reviewed.

Open access This is an open access article distributed in accordance with the Creative Commons Attribution Non Commercial (CC BY-NC 4.0) license, which permits others to distribute, remix, adapt, build upon this work non-commercially, and license their derivative works on different terms, provided the original work is properly cited, appropriate credit is given, any changes made indicated, and the use is non-commercial. See: http://creativecommons.org/licenses/by-nc/4.0/.

\section{ORCID iDs}

Mandy M Archibald http://orcid.org/0000-0003-4767-1031

Roberta L Woodgate http://orcid.org/0000-0002-7176-2390

\section{REFERENCES}

1 Canadian Institutes of Health Research. Knowledge translation at CIHR, 2016. Available: http://www.cihr-irsc.gc.ca/e/29418.html\#1

2 Greenhalgh T, Humphrey C, Woodard F. User involvement in health care. Chichester, West Sussex, UK: Wiley-Blackwell/BMJ Books, 2011.

3 Graham ID, Kothari A, McCutcheon C, et al. Moving knowledge into action for more effective practice, programmes and policy: protocol for a research programme on integrated knowledge translation. Implement Sci 2018;13:22-15.

4 Archibald MM, Lawless M, Harvey G, et al. Transdisciplinary research for impact: protocol for a realist evaluation of the relationship between transdisciplinary research collaboration and knowledge translation. BMJ Open 2018;8:e021775.

5 Palmer VJ, Weavell W, Callander R, et al. The participatory Zeitgeist: an explanatory theoretical model of change in an era of coproduction and codesign in healthcare improvement. Med Humanit 2019;45:247-57.

6 Ocloo J, Matthews R. From tokenism to empowerment: progressing patient and public involvement in healthcare improvement. BMJ Qual Saf 2016;25:626-32

7 Archibald MM, Scott SD. Learning from usability testing of an artsbased knowledge translation tool for parents of a child with asthma. Nurs Open 2019;6:1615-25.

8 Archibald MM, Hartling L, Ali S, et al. Developing "My Asthma Diary": a process exemplar of a patient-driven arts-based knowledge translation tool. BMC Pediatr 2018:18:186.

9 Shimmin C, Wittmeier KDM, Lavoie JG, et al. Moving towards a more inclusive patient and public involvement in health research paradigm: the incorporation of a trauma-informed intersectional analysis. BMC Health Serv Res 2017;17:539.

10 Canadian Institutes of Health Research. Integrating ethics and the knowledge to action cycle, 2018. http://www.cihr-irsc.gc.ca/e/48802. html

11 European network of living Labs, 2019. Available: https://enoLiving lab.org/about-us/

12 French M, Miller FA. Leveraging the "living laboratory": on the emergence of the entrepreneurial hospital. Soc Sci Med 2012;75:717-24.

13 Martin C. Main motivation for installation of new living laboratory for health. 17th Int. Conf. on E-Health Networking, Application \& Services (HealthCom), 2015:160-3.

14 Hossain M, Leminen S, Westerlund M. A systematic review of living lab literature. J Clean Prod 2019;213:976-88.

15 Bergvall-Kareborn B, Hoist M, Stahlbrost A. Concept design with a living lab approach. 2009 42nd Hawaii International Conference on System Sciences, 2009:1-10.

16 Guimont D, Lapointe D. Empowering local tourism providers to innovate through a living lab process: does scale matter? Technology Innovation Management Review 2016;6:18-25.

17 Ståhlbröst A, Holst M. The Living lab Methodology Handbook. Soc Informatics Luleå Univ Technol CDT - Cent Distance-spanning Technol, 2012: 76. http://www.Itu.se/cms_fs/1.101555!/file/LivingLa bsMethodologyBook web.pdf

$18 \mathrm{Kim}$ J, Kim YL, Jang $\vec{H}$. Living Labs for health: an integrative literature review. Eur J Public Health 2019;30:1-9.
19 Malmberg K, Vaittinen I, Ståhlbröst A. D2.2 living Labs methodology Handbook. ENoLL, 2017: 1-56. https://u4iot.eu/pdf/D2.2_LivingLa bsMethodologyHandbook.pdf

20 Folstad A. Living Labs for innovation and development of information and communication technology: a literature review. Electronic Journal of Virtual Organizations 2008;10:99-131.

21 Schuurman D, De Marez L, Ballon P. Living Labs: a systematic literature review. Open Living Lab Days 2015, Proceedings. Presented at the Open Living Lab Days 2015, 2015.

22 Noublanche F, Jaglin-Grimonprez C, Sacco G, et al. The development of gerontechnology for hospitalized frail elderly people: the ALLEGRO hospital-based geriatric living lab. Maturitas 2019;125:17-19.

23 Vallentin-Holbech L, Dalgaard Guldager J, Dietrich T, et al. Cocreating a virtual alcohol prevention simulation with young people. Int J Environ Res Public Health 2020;17:1097.

24 Boman I-L, Lundberg S, Starkhammar S, et al. Exploring the usability of a videophone mock-up for persons with dementia and their significant others. BMC Geriatr 2014;14:49.

25 Kanstrup AM. Living in the lab: an analysis of the work in eight living laboratories set up in care homes for technology innovation. CoDesign 2017;13:49-64.

26 Leminen S, Westerlund M, Nyström A-G. Living Labs as OpenInnovation networks. Technol Innov Manag Rev 2012;2:6-11.

27 Creswell JW, Plano Clark VL. Designing and conducting mixed methods research. thousand oak. CA: Sage, 2017.

28 Thorne S. Interpretive description. Walnut Creek, CA: Left Coast Press, 2008.

29 Angell C, Alexander J, Hunt JA. 'Draw, write and tell': A literature review and methodological development on the 'draw and write' research method. J Early Child Res 2015;13:17-28.

30 Kitson A, Powell K, Hoon E, et al. Knowledge translation within a population health study: how do you do it? Implementation Sci 2013;8:54.

31 Liu M, Wronski L. Examining completion rates in web surveys via over 25,000 real-world surveys. Soc Sci Comput Rev 2018;36:116-24.

32 Dillman DA, Smyth JD. Design effects in the transition to web-based surveys. Am J Prev Med 2007;32:S90-6.

33 Aerny-Perreten N, Domínguez-Berjón MF, Esteban-Vasallo MD, et al. Participation and factors associated with late or non-response to an online survey in primary care. J Eval Clin Pract 2015;21:688-93.

34 Saleh A, Bista K. Examining factors impacting online survey response rates in educational research: perceptions of graduate students. J Multidiscip Eval 2017;13:63-74.

35 Archibald M, Scott S, Hartling L. Mapping the waters: a scoping review of the use of visual arts in pediatric populations with health conditions. Arts Health 2014;6:5-23.

36 Archibald MM, Ambagtsheer RC, Casey MG, et al. Using Zoom videoconferencing for qualitative data collection: perceptions and experiences of researchers and participants. Int J Qual Methods 2019;18:160940691987459.

37 Archibald MM, Ambagtsheer R, Beilby J, et al. Perspectives of frailty and frailty screening: protocol for a collaborative knowledge translation approach and qualitative study of Stakeholder understandings and experiences. BMC Geriatr 2017;17:87.

38 Luthy C, Cedraschi C, Pasquina P, et al. Perception of chronic respiratory impairment in patients' drawings. J Rehabil Med 2013;45:694-700.

39 Guetterman TC, Fetters MD, Creswell JW. Integrating quantitative and qualitative results in health science mixed methods research through joint displays. Ann Fam Med 2015;13:554-61.

40 Archibald MM, Lawless M, Gill TK, et al. Orthopaedic surgeons' perceptions of frailty and frailty screening. BMC Geriatr 2020;20:1-11.

41 Kitson A, Brook A, Harvey G, et al. Using complexity and network concepts to inform healthcare knowledge translation. Int $\mathrm{J}$ Health Policy Manag 2018;7:231-43.

42 Morris ZS, Wooding S, Grant J. The answer is 17 years, what is the question: understanding time lags in translational research. J $R$ Soc Med 2011;104:510-20.

43 Archibald MM, Caine V, Scott SD. The development of a classification schema for arts-based approaches to knowledge translation. Worldviews Evid Based Nurs 2014;11:316-24. 